\title{
Synchronizing Road Coloring
}

\author{
A.N. Trahtman* ${ }^{\dagger}$ \\ Bar-Ilan University, Dep. of Math., 52900, Ramat Gan, Israel
}

\begin{abstract}
The synchronizing word of a deterministic automaton is a word in the alphabet of colors (considered as letters) of its edges that maps the automaton to a single state. A coloring of edges of a directed graph is synchronizing if the coloring turns the graph into a deterministic finite automaton possessing a synchronizing word.

The road coloring problem is the problem of synchronizing coloring of a directed finite strongly connected graph with constant outdegree of all its vertices if the greatest common divisor of lengths of all its cycles is one. The problem was posed by Adler, Goodwyn and Weiss over 30 years ago and evoked noticeable interest among the specialists in the theory of graphs, finite automata, coding and symbolic dynamics. Many partial solutions of the problem have been found and different generalizations were considered.

The positive solution of the road coloring problem is presented below. We reproduce from the literature also the statements used in our proof. The necessary and sufficient conditions of synchronizing road coloring of directed graph with constant outdegree of a vertex are presented.
\end{abstract}

Key words: road coloring problem, graph, deterministic finite automaton, synchronization.

\section{Introduction}

The road coloring problem originates in [2] and was stated explicitly in [1] for a strongly connected directed finite graph with constant outdegree of all its vertices where the greatest common divisor ( gcd) of lengths of all its cycles is one. The edges of the graph are unlabelled. The task is to find a labelling of the edges that turns the graph into a deterministic finite automaton possessing a synchronizing word. So the road coloring problem is connected with the problem of existence of synchronizing word for deterministic complete finite automaton. The condition on gcd is necessary [1], [5]. It can be replaced by the equivalent property that there does not exist a partition of the set of vertices on subsets $V_{1}, V_{2}, \ldots, V_{k}=V_{1}(k>1)$ such that every edge which begins in $V_{i}$ has its end in $V_{i+1}$ [5], [14]. The outdegree of the vertex can be considered also as the size of an alphabet where the letters denote colors.

The road coloring problem is important in automata theory: a synchronizing

\footnotetext{
* Email: trakht@macs.biu.ac.il

$\dagger$ http://www.cs.biu.ac.il/ trakht/syn.html
}

Please use the following format when citing this chapter:

Trahtman, A.N., 2008, in IFIP International Federation for Information Processing, Volume 273; Fifth IFIP International Conference on Theoretical Computer Science; Giorgio Ausiello, Juhani Karhumäki, Giancarlo Mauri, Luke Ong; (Boston: Springer), pp. 43-53. 
coloring makes the behavior of an automaton resistant against input errors since, after detection of an error, a synchronizing word can reset the automaton back to its original state, as if no error had occurred. The problem appeared first in the context of symbolic dynamics and is important also in this area.

Together with the Cerny conjecture, the road coloring problem belongs to the most fascinating problems in the theory of finite automata [13], [16], [17]. The problem is discussed even in "Wikipedia" - the popular Internet Encyclopedia. However, at the same time it proved to be hard and was considered as a "notorious open problem" [12], [5] and "unfeasible" [8].

Several partial solutions in this area have been found within last thirty years. In [14] it is shown that a graph with no multiple edges (i.e. no distinct edges in $\mathrm{G}$ have the same source and the same target) and with a simple cycle of prime length has a synchronizing coloring. In [6] it is shown that a graph of outdegree two with a simple cycle of length relatively prime to the weight of the graph (i.e. the sum of the components of an integer Perron left eigenvector chosen with relatively prime components) has a synchronizing coloring. The conjecture is true for Eulerian digraphs [10] (i.e. the indegree of any vertex is equal to the outdegree). In [5] the problem is solved for the class of automata having always stable synchronizing pair of states. The class is closed under some kind of homomorphism. The conjecture has positive solution also if the outdegree of vertices is relatively great [7]. Another special case, proven in [15], is that a graph with all vertices of indegree 1 except one (these graphs are trees where all leaves merge with the root), has a synchronizing coloring. In [9] it is shown that a graph of outdegree $k$ which is decomposable in $k$ disjoint monochromatic subgraphs containing exactly one cycle, has a synchronizing coloring if the greater common divisor of the lengths of the monochromatic cycles equals 1 . The last result was strengthened in [4] for strongly disjoint set of cycles. The structure theory of the minimal ideal of a finite semigroup plays an essential role in [3]. The concept from [6] of the weight of a vertex supposed by Friedman and the concept of a stable pair of states of Culik, Karhumaki and Kari [5], [10] with corresponding results and consequences are essentially used in our proof. We also reproduce from the literature the proofs of some related statements for to complete the picture.

The road coloring conjecture is settled in the affirmative: a finite directed strongly connected graph with constant outdegree of all vertices has a synchronizing coloring iff the great common divisor of the lengths of all its cycles is equal to one.

The necessary and sufficient conditions of synchronizing road coloring of directed graph with constant outdegree of a vertex are presented. 


\section{Preliminaries}

A finite directed strongly connected graph with constant outdegree of all its vertices where the gcd of lengths of all its cycles is one will be called $A G W$ graph as aroused by Adler, Goodwyn and Weiss.

If there exists a path in an automaton from the state $\mathbf{p}$ to the state $\mathbf{q}$ and the edges of the path are consecutively labeled by $\sigma_{1}, \ldots, \sigma_{k}$, then for $s=\sigma_{1} \ldots \sigma_{k} \in$ $\Sigma^{+}$let us write $\mathbf{q}=\mathbf{p} s$.

Let $P s$ be the map of the subset $P$ of states of an automaton by help of $s \in \Sigma^{+}$ and let $P s^{-1}$ be the maximal set of states $Q$ such that $Q s \subseteq P$. For the transition graph $\Gamma$ of an automaton let $\Gamma s$ denote the map of the set of states of the automaton.

$|P|$ - the size of the subset $P$ of states from an automaton (of vertices from a graph).

A word $s \in \Sigma^{+}$is called a synchronizing word of the automaton with transition graph $\Gamma$ if $|\Gamma s|=1$.

A coloring of a directed finite graph is synchronizing if the coloring turns the graph into a deterministic finite automaton possessing a synchronizing word.

A pair of distinct states $\mathbf{p}, \mathbf{q}$ of an automaton (of vertices of the transition graph) will be called synchronizing if $\mathbf{p} s=\mathbf{q} s$ for some $s \in \Sigma^{+}$. In the opposite case, if for any $s \mathbf{p} s \neq \mathbf{q} s$, we call the pair deadlock.

A synchronizing pair of states $\mathbf{p}, \mathbf{q}$ of an automaton is called stable if for any word $u$ the pair $\mathbf{p} u, \mathbf{q} u$ is also synchronizing [5], [10].

We call the set of all outgoing edges of a vertex a bunch if all these edges are incoming edges of only one vertex.

Let $u$ be a left eigenvector with positive components having no common divisor of adjacency matrix of a graph with vertices $\mathbf{p}_{1}, \ldots, \mathbf{p}_{n}$. The i-th component $u_{i}$ of the vector $u$ is called the weight of the vertex $\mathbf{p}_{i}$ and denoted by $w\left(\mathbf{p}_{i}\right)$. The sum of the weights of the vertices from a set $D$ is denoted by $w(D)$ and is called the weight of $D[6]$.

The subset $D$ of states of an automaton (of vertices of the transition graph $\Gamma$ of the automaton) such that $w(D)$ is maximal and $|D s|=1$ for some word $s \in \Sigma^{+}$ let us call $F$-maximal as introduced by Friedman [6].

The subset $\Gamma s$ of states (of vertices of the transition graph $\Gamma$ ) for some word $s$ such that every pair of states from the set is deadlock will be called an F-clique.

\section{Some properties of $\boldsymbol{F}$-clique and of coloring free of stable pairs}

The road coloring problem was formulated for $A G W$ graphs [1], [2] and only such graphs are considered below. The primitive cases of graphs with loops and of only one color can be also omitted [1], [14]. Let us formulate some important 
results from [6], [5] and [10] together with some useful statements [1], [14] in the following form:

Theorem 1 [6] There exists a partition of $\Gamma$ on $F$-maximal sets (of the same weight).

Proof. Let $\Gamma$ have outdegree $d$ everywhere. The vector $e=(1, \ldots, 1)$ is a right eigenvector with eigenvalue $d$ of the adjacency matrix [11] $A$ of $\Gamma$. Since $\Gamma$ is strongly connected, by Perron-Frobenius Theorem [11] the matrix $A$ with non-negative elements has a positive left eigenvector $w=\left(w_{1}, \ldots, w_{n}\right)$ of integer components with the same eigenvalue $d$, i.e. $w A=d w$. The component $w_{i}$ of the vector $w$ is defined as the weight $w\left(\mathbf{p}_{i}\right)>0$ of the state $\mathbf{p}_{i}$.

Let $\mathbf{q}$ be arbitrary state and $Q$ be the set of states $q \sigma^{-1}$ for all $\sigma \in \Sigma$. Then $\sum_{\mathbf{r} \in Q} w(\mathbf{r})=d w(\mathbf{q})$ because $w A=d w$. Consequently for any set $R$ of states $d w(R)=\sum_{\sigma \in \Sigma} R \sigma^{-1}$. It implies, in particular, that from $w(R)>w\left(R \sigma^{-1}\right)$ for some $\sigma \in \Sigma$ follows $w(R)<w\left(R \alpha^{-1}\right)$ for some another $\alpha \in \Sigma$. Therefore for $F$-maximal set $R$ holds $w(R)=w\left(R \sigma^{-1}\right)$ for any $\sigma$ and $w(R)=w\left(R s^{-1}\right)$ for any $s \in \Sigma^{+}$.

For $F$-maximal set $R$ and some word $s|R s|=1$. Since $\Gamma$ is strongly connected, for any state $\mathbf{p}$ there exists a word $t=t_{1} s$ such that $R t=\mathbf{p}$. So for any state $\mathbf{p}$ and some word $t$ the set of states $\mathbf{p} t^{-1}$ is $F$-maximal. For any state $\mathbf{r} \notin \mathbf{p} t^{-1}$ and some word $u$ is also $F$-maximal. The set $\mathbf{p} t^{-1} u^{-1}$ is $F$-maximal, too. Both obtained $F$-maximal sets $\mathbf{p} t^{-1} u^{-1}$ and $\mathbf{r} u^{-1}$ are disjoint. The continuation of this process for states outside obtained $F$-maximal sets gives us a partition of $\Gamma$ on $F$-maximal sets.

Lemma 1 [1], [5], [14]. Let $\Gamma$ be directed graph. Then the greatest common divisor of lengths of all its cycles is $k$ if and only if there exists a partition of the set of vertices on subsets $V_{1}, V_{2}, \ldots, V_{k+1}=V_{1}$ such that every edge which begins in $V_{i}$ has its end in $V_{i+1}$.

Proof. Indeed, in the case of such partition of size $k>1$ the length of any cycle of the graph is divided by $k$.

In the case $k$ is a common divisor of length of all cycles of the graph let us enumerate the vertices of the graph. We begin from an arbitrary vertex and suppose $n(\mathbf{q})=n(\mathbf{p})+1$ (modulo $k$ ) if there exist an edge $\mathbf{p} \rightarrow \mathbf{q}$. The contradiction in the enumeration is impossible because the difference between length of cycles is divided by $k$. Then suppose $\mathbf{q} \in V_{m}$ if $n(\mathbf{q})=m$ (modulo $k$ ). Therefore every edge which begins in $V_{i}$ ends in $V_{i+1}$. So the desired partition exists.

Let us recall that a binary relation $\rho$ on the set of the states of an automaton is called congruence if $\rho$ is equivalence and for any word $u$ from $\mathbf{p} \rho \mathbf{q}$ follows $\mathbf{p} u \rho \mathbf{q} u$.

Theorem 2 [5], [10] Let us consider a coloring of AGW graph $\Gamma$. Stability of states is a binary relation on the set of states of the obtained automaton; denote this relation by $\rho$. Then $\rho$ is a congruence relation, $\Gamma / \rho$ presents an $A G W$ graph and synchronizing coloring of $\Gamma / \rho$ implies synchronizing recoloring of $\Gamma$. 
Proof. Suppose $\mathbf{p} \rho \mathbf{q}$ and $\mathbf{q} \rho \mathbf{r}$. Then for any word $u$ there exists a word $s$ such that $\mathbf{p} u s=\mathbf{q} u s$. The couple of states $\mathbf{q}, \mathbf{r}$ is stable, whence there exists a word $t$ such that for arbitrary $u \mathbf{q} u s t=\mathbf{r} u s t$. So for any $u$ there exists a word $s t$ such that $\mathbf{p} u s t=\mathbf{r}$ ust. Hence $\mathbf{p} \rho \mathbf{r}$ and the relation $\rho$ is transitive and stable. It implies the equivalence of $\rho$. From $\mathbf{p} \rho \mathbf{q}$ follows $\mathbf{p} s \rho \mathbf{q} s$ for any $s$ (because the pair $\mathbf{p} s, \mathbf{q} s$ is also stable) and therefore the relation $\rho$ is a congruence.

The outdegree of a state in the quotient automaton $\Gamma / \rho$ is equal to the same number of colors as in $\Gamma, \Gamma / \rho$ is strongly connected just as $\Gamma$.

The condition on $\mathrm{gcd}$ can be replaced by the equivalent property that there does not exist a partition of the set of vertices on subsets $V_{1}, V_{2}, \ldots, V_{k}=V_{1}$ such that every edge which begins in $V_{i}$ has its end in $V_{i+1}$ (Lemma 1).

The non-trivial such partition of $\Gamma / \rho$ exists only if $\Gamma$ has also such partition. Every edge with beginning in image of $V_{i}$ has its end in image of $V_{i+1}$. Therefore the condition on $g c d$ is stable, whence $\Gamma / \rho$ is $A G W$ graph.

Suppose now that $\Gamma / \rho$ has a synchronizing coloring. The synchronizing coloring of $\Gamma / \rho$ induces a synchronizing coloring of the original automaton as follows: we color all the preimages of an edge of $\Gamma / \rho$ by the same color. For any pair of states from $\Gamma$ the synchronizing word of the images of the states in $\Gamma / \rho$ takes both states into one equivalence class of the relation $\rho$ on $\Gamma$. Any couple of states from this class is stable and therefore synchronizing. So via such coloring any pair of states from $\Gamma$ is synchronizing.

The last theorem shows that if every $A G W$ graph has a coloring with a stable pair, then every $A G W$ graph has a synchronizing coloring. So the problem is reduced to the search of a coloring with stable pair.

Lemma 2 Let $w$ be the weight of F-maximal set of the AGW graph $\Gamma$ via some coloring. Then the size of every F-clique of the coloring is the same and equal to $w(\Gamma) / w$ (the size of partition of $\Gamma$ on $F$-maximal sets).

Proof. Two states from an $F$-clique could not belong to one $F$-maximal set because this pair is not synchronizing. By Theorem 1 there exists a partition of $\Gamma$ on $F$-maximal sets of weight $w$. So the partition consists from $w(\Gamma) / w$ $F$-maximal sets and to every $F$-maximal set belongs at most one state from $F$-clique. Consequently, the size of any $F$-clique is not greater than $w(\Gamma) / w$.

Let $\Gamma s$ be an $F$-clique. The sum of the weights $\mathbf{q} s^{-1}$ for all $\mathbf{q} \in \Gamma s$ is the weight of $\Gamma$. So

$$
w(\Gamma)=\sum_{q \in \Gamma s} w\left(\mathbf{q} s^{-1}\right)
$$

The number of addends (the size of the $F$-clique) is not greater than $w(\Gamma) / w$. The weight of the set $\mathbf{q} s^{-1}$ for every $\mathbf{q} \in \Gamma s$ is not greater than $w$. Therefore $\mathbf{q} s^{-1}$ is an $F$-maximal set of weight $w$ for every $\mathbf{q} \in \Gamma s$ and the size of any $F$ clique is $w(\Gamma) / w$, the number of $F$-maximal sets in the corresponding partition of $\Gamma$. 
Lemma 3 Let $F$ be $F$-clique via some coloring of $A G W$ graph $\Gamma$. For any word $s$ the set Fs is also an F-clique and any state [vertex] $\mathbf{p}$ belongs to some F-clique.

Proof. Any pair $\mathbf{p}, \mathbf{q}$ from an $F$-clique $F$ is a deadlock. To be deadlock is a stable binary relation, therefore for any word $s$ the pair $\mathbf{p} s, \mathbf{q} s$ from $F s$ also is a deadlock. So all pairs from $F s$ are deadlocks.

For the $F$-clique $F$ there exists a word $t$ such that $\Gamma t=F$. Thus $\Gamma t s=F s$, whence $F s$ is an $F$-clique.

For any $\mathbf{r}$ from a strongly connected graph $\Gamma$, there exists a word $\mathrm{u}$ such that $\mathbf{r}=\mathbf{p} u$ for $\mathbf{p}$ from the $F$-clique $F$, whence $\mathbf{r}$ belongs to the $F$-clique $F u$.

Lemma 4 Let $A$ and $B(|A|>1)$ be distinct $F$-cliques via some coloring without stable pairs of the $A G W$ graph $\Gamma$. Then $|A|-|A \cap B|=|B|-|A \cap B|>1$.

Proof. Let us assume the contrary: $|A|-|A \cap B|=1$. By Lemma $2,|A|=|B|$. So $|B|-|A \cap B|=1$, too. The pair of states $\mathbf{p} \in A \backslash B$ and $\mathbf{q} \in B \backslash A$ is not stable. Therefore for some word $s$ the pair $(\mathbf{p} s, \mathbf{q} s)$ is a deadlock. Any pair of states from the $F$-clique $A$ and from the $F$-clique $B$ as well as from $F$-cliques $A s$ and $B s$ is a deadlock. So any pair of states from the set $(A \cup B) s$ is a deadlock. One has $|(A \cup B) s|=|A|+1>|A|$.

In view of Theorem 1 , there exists a partition of size $|A|$ (Lemma 2) of $\Gamma$ on $F$ maximal sets. To every $F$-maximal set belongs at most one state from $(A \cup B) s$ because every pair of states from this set is a deadlock and no deadlock could belong to an $F$-maximal set. This contradicts the fact that the size of $(A \cup B) s$ is greater than $|A|$.

Lemma 5 Let some vertex of $A G W$ graph $\Gamma$ have two incoming bunches. Then any coloring of $\Gamma$ has a stable couple.

Proof. If a vertex $\mathbf{p}$ has two incoming bunches from vertices $\mathbf{q}$ and $\mathbf{r}$, then the couple $\mathbf{q}, \mathbf{r}$ is stable for any coloring because $\mathbf{q} \alpha=\mathbf{r} \alpha=\mathbf{p}$ for any letter (color) $\alpha \in \Sigma$.

\section{The spanning subgraph of cycles and trees with maximal number of edges in the cycles}

Définition 1 Let us call a subgraph $S$ of the $A G W$ graph $\Gamma$ a spanning subgraph of $\Gamma$ if to $S$ belong all vertices of $\Gamma$ and exactly one outgoing edge of every vertex.

A maximal subtree of the spanning subgraph $S$ with root on a cycle from $S$ and having no common edges with cycles from $S$ is called a tree of $S$.

The length of path from a vertex $\mathbf{p}$ through the edges of the tree of the spanning set $S$ to the root of the tree is called the level of $\mathbf{p}$ in $S$. 
Remark 1 Any spanning subgraph $S$ consists of disjoint cycles and trees with roots on cycles; any tree and cycle of $S$ is defined identically, the level of the vertex from cycle is zero, the vertices of trees except root have positive level, the vertex of maximal positive level has no incoming edge from $S$. The edges of every given color by any coloring form a spanning subgraph and for any spanning subgraph there exists a corresponding coloring.

Lemma 6 Let $N$ be a set of vertices of level $n$ from some tree of the spanning subgraph $S$ of $A G W$ graph $\Gamma$. Then in a coloring of $\Gamma$ where all edges of $S$ have the same color $\alpha$, any $F$-clique $F$ satisfies $|F \cap N| \leq 1$.

Proof. Some power of $\alpha$ synchronizes all states of given level of the tree and maps them into the root. Any couple of states from an $F$-clique could not be synchronized and therefore could not belong to $N$.

Lemma 7 Let $A G W$ graph $\Gamma$ have a spanning subgraph $R$ of only disjoint cycles (without trees). Then $\Gamma$ also has another spanning subgraph with exactly one vertex of maximal positive level.

Proof. The spanning subgraph $R$ has only cycles and therefore the levels of all vertices are equal to zero. In view of $g c d=1$ in the strongly connected graph $\Gamma$, not all edges belong to a bunch. Therefore there exist two edges $u=\mathbf{p} \rightarrow \mathbf{q} \notin R$ and $v=\mathbf{p} \rightarrow \mathbf{s} \in R$ with common first vertex $\mathbf{p}$ but such that $\mathbf{q} \neq \mathbf{s}$. Let us replace the edge $v=\mathbf{p} \rightarrow \mathbf{s}$ from $R$ by $u$. Then only the vertex $\mathbf{s}$ has maximal level $L>0$ in the new spanning subgraph.

Lemma 8 Let any vertex of an $A G W$ graph $\Gamma$ have no two incoming bunches. Then $\Gamma$ has a spanning subgraph such that all its vertices of maximal positive level belong to one non-trivial tree.

Let us consider a spanning subgraph $R$ with a maximal number of vertices [edges] in its cycles. In view of Lemma 7, suppose that $R$ has non-trivial trees and let $L>0$ be the maximal value of the level of a vertex.

Further consideration is necessary only if at least two vertices of level $L$ belong to distinct trees of $R$ with distinct roots.

Let us consider a tree $T$ from $R$ with vertex $\mathbf{p}$ of maximal level $L$ and edge $\bar{b}$ from vertex $\mathbf{b}$ to the tree root $\mathbf{r} \in T$ on the path of length $L$ from $\mathbf{p}$. Let the root $\mathbf{r}$ belong to the cycle $H$ of $R$ with the edge $\bar{c}=\mathbf{c} \rightarrow \mathbf{r} \in H$. There exists also an edge $\bar{a}=\mathbf{a} \rightarrow \mathbf{p}$ that does not belong to $R$ because $\Gamma$ is strongly connected and $\mathbf{p}$ has no incoming edge from $R$.

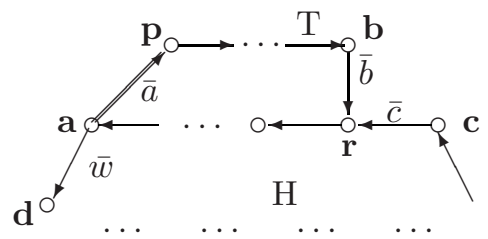

We consider the path in $T$ from $\mathbf{p}$ to $\mathbf{r}$ of maximal length $L$. Our aim is to 
extend the maximal level of the vertex on the extension of the tree $T$ much more than the maximal level of vertex of other trees from $R$. We plan to use the following three changes:

1) replace the edge $\bar{w}$ from $R$ with first vertex a by the edge $\bar{a}=\mathbf{a} \rightarrow \mathbf{p}$,

2 ) replace the edge $\bar{b}$ from $R$ by some other outgoing edge of the vertex $\mathbf{b}$,

3 ) replace the edge $\bar{c}$ from $R$ by some other outgoing edge of the vertex $\mathbf{c}$.

If one of the ways does not succeed let us go to the next assuming the situation in which the previous way fails and excluding the successfully studied cases. So we diminish the considered domain. We can use sometimes two changes together. Let us begin with

1) Suppose first $\mathbf{a} \notin H$. If $\mathbf{a}$ belongs to a path in $T$ from $\mathbf{p}$ to $\mathbf{r}$ then a new cycle with part of the path and edge $\mathbf{a} \rightarrow \mathbf{p}$ is added to $R$ extending the number of vertices in its cycles in spite of the choice of $R$. In opposite case the level of a in the new spanning subgraph is $L+1$ and the vertex $\mathbf{r}$ is a root of the new tree containing all vertices of maximal level (in particular, the vertex a or its ancestors in $\mathrm{R}$ ).

So let us assume $\mathbf{a} \in H$ and suppose $\bar{w}=\mathbf{a} \rightarrow \mathbf{d} \in H$. In this case the vertices $\mathbf{p}, \mathbf{r}$ and a belong to a cycle $H_{1}$ with new edge $\bar{a}$ of a new spanning subgraph $R_{1}$. So we have the cycle $H_{1} \in R_{1}$ instead of $H \in R$. If the length of path from $\mathbf{r}$ to $\mathbf{a}$ in $H$ is $r_{1}$ then $H_{1}$ has length $L+r_{1}+1$. A path to $\mathbf{r}$ from the vertex $\mathbf{d}$ of the cycle $H$ remains in $R_{1}$. Suppose its length is $r_{2}$. So the length of the cycle $H$ is $r_{1}+r_{2}+1$. The length of the cycle $H_{1}$ is not greater than the length of $H$ because the spanning subgraph $R$ has maximal number of edges in its cycles. So $r_{1}+r_{2}+1 \geq L+r_{1}+1$, whence $r_{2} \geq L$. If $r_{2}>L$, then the length $r_{2}$ of the path from $\mathbf{d}$ to $\mathbf{r}$ in a tree of $R_{1}$ (and the level of $\mathbf{d}$ ) is greater than $L$ and the level of $\mathbf{d}$ (or of some other ancestor of $\mathbf{r}$ in a tree from $R$ ) is the desired unique maximal level.

So assume for further consideration $L=r_{2}$ and $\mathbf{a} \in H$. Analogously, for any vertex of maximal level $L$ with root in the cycle $H$ and incoming edge from a vertex $\mathbf{a}_{1}$ the proof can be reduced to the case $\mathbf{a}_{1} \in H$ and $L=r_{2}$ for the corresponding new value of $r_{2}$.

2) Suppose the set of outgoing edges of the vertex $\mathbf{b}$ is not a bunch. So one can replace in $R$ the edge $\bar{b}$ from the vertex $\mathbf{b}$ by an edge $\bar{v}$ from $\mathbf{b}$ to a vertex $\mathbf{v} \neq \mathbf{r}$.

The vertex $\mathbf{v}$ could not belong to $T$ because in this case a new cycle is added to $R$ and therefore a new spanning subgraph has a number of vertices in the cycles greater than in $R$.

If the vertex $\mathbf{v}$ belongs to another tree of $R$ but not to cycle, then $T$ is a part of a new tree $T_{1}$ with a new root of a new spanning subgraph $R_{1}$ and the path from $\mathbf{p}$ to the new root is extended. So only the tree $T_{1}$ has states of new maximal level.

If $\mathbf{v}$ belongs to some cycle $H_{2} \neq H$ from $R$, then together with replacing $\bar{b}$ by $\bar{v}$, we replace also the edge $\bar{w}$ by $\bar{a}$. So we extend the path from $\mathbf{p}$ to the new root $\mathbf{v}$ at least by the edge $\bar{a}=\mathbf{a} \rightarrow \mathbf{p}$ and by almost all edges of $H$. Therefore the new maximal level $L_{1}>L$ has either the vertex $\mathbf{d}$ or its ancestors from the 
old spanning subgraph $R$.

Now there remains only the case when $\mathbf{v}$ belongs to the cycle $H$. The vertex $\mathbf{p}$ also has level $L$ in new tree $T_{1}$ with root $\mathbf{v}$. The only difference between $T$ and $T_{1}$ (just as between $R$ and $R_{1}$ ) is the root and the incoming edge of the root. The new spanning subgraph $R_{1}$ has also a maximal number of vertices in cycles just as $R$. Let $r_{3}$ be the length of the path from $\mathbf{d}$ to the new root $\mathbf{v} \in H$.

For the spanning subgraph $R_{1}$, one can obtain $L=r_{3}$ just as it was done on the step 1) for $R$. From $\mathbf{v} \neq \mathbf{r}$ follows $r_{3} \neq r_{2}$, though $L=r_{3}$ and $L=r_{2}$.

So for further consideration suppose that the set of outgoing edges of the vertex $\mathbf{b}$ is a bunch to $\mathbf{r}$.

3) The set of outgoing edges of the vertex $\mathbf{c}$ is not a bunch to $\mathbf{r}$ because $\mathbf{r}$ has another bunch from $\mathbf{b}$.

Let us replace in $R$ the edge $\bar{c}$ by an edge $\bar{u}=\mathbf{c} \rightarrow \mathbf{u}$ such that $\mathbf{u} \neq \mathbf{r}$. The vertex $\mathbf{u}$ could not belong to the tree $T$ because in this case the cycle $H$ is replaced by a cycle with all vertices from $H$ and some vertices of $T$ whence its length is greater than $|H|$. Therefore the new spanning subgraph has a number of vertices in its cycles greater than in spanning subgraph $R$ in spite of the choice of $R$.

So remains the case $\mathbf{u} \notin T$. Then the tree $T$ is a part of a new tree with a new root and the path from $\mathbf{p}$ to the new root is extended at least by a part of $H$ from the former root $\mathbf{r}$. The new level of $\mathbf{p}$ therefore is maximal and greater than the level of any vertex in some another tree.

Thus anyway there exists a spanning subgraph with vertices of maximal level in one non-trivial tree.

Theorem 3 Any AGW graph $\Gamma$ has coloring with stable couples.

Proof. By Lemma 5, in the case of vertex with two incoming bunches $\Gamma$ has a coloring with stable couples. In opposite case, by Lemma $8, \Gamma$ has a spanning subgraph $R$ such that the vertices of maximal positive level $L$ belong to one tree of $R$.

Let us give to the edges of $R$ the color $\alpha$ and denote by $C$ the set of all vertices from the cycles of $R$. Then let us color the remaining edges of $\Gamma$ by other colors arbitrarily.

By Lemma 3 , in a strongly connected graph $\Gamma$ for every word $s$ and $F$-clique $F$ of size $|F|>1$, the set $F s$ also is an $F$-clique (of the same size by Lemma 2) and for any state $\mathbf{p}$ there exists an $F$-clique $F$ such that $\mathbf{p} \in F$.

In particular, some $F$ has non-empty intersection with the set $N$ of vertices of maximal level $L$. The set $N$ belongs to one tree, whence by Lemma 6 this intersection has only one vertex. The word $\alpha^{L-1}$ maps $F$ on an $F$-clique $F_{1}$ of size $|F|$. One has $\left|F_{1} \backslash C\right|=1$ because the sequence of edges of color $\alpha$ from any tree of $R$ leads to the root of the tree, the root belongs to a cycle colored by $\alpha$ from $C$ and only for the set $N$ with vertices of maximal level holds $N \alpha^{L-1} \nsubseteq C$. So $\left|N \alpha^{L-1} \cap F_{1}\right|=\left|F_{1} \backslash C\right|=1$ and $\left|C \cap F_{1}\right|=\left|F_{1}\right|-1$.

Let the integer $m$ be a common multiple of the lengths of all considered cycles from $C$ colored by $\alpha$. So for any $\mathbf{p}$ from $C$ as well as from $F_{1} \cap C$ holds $\mathbf{p} \alpha^{m}=\mathbf{p}$. 
Therefore for an $F$-clique $F_{2}=F_{1} \alpha^{m}$ holds $F_{2} \subseteq C$ and $C \cap F_{1}=F_{1} \cap F_{2}$.

Thus two $F$-cliques $F_{1}$ and $F_{2}$ of size $\left|F_{1}\right|>1$ have $\left|F_{1}\right|-1$ common vertices. So $\left|F_{1} \backslash\left(F_{1} \cap F_{2}\right)\right|=1$. Consequently, in view of Lemma 4 , there exists a stable couple in the considered coloring.

Theorem 4 Every AGW graph $\Gamma$ has synchronizing coloring.

The proof follows from Theorems 3 and 2 .

\section{The necessary and sufficient conditions of synchronizing coloring of an arbitrary graph}

Theorem 5 Let every vertex of strongly connected directed finite graph $\Gamma$ have the same number of outgoing edges. Then $\Gamma$ has synchronizing coloring if and only if the greatest common divisor of lengths of all its cycles is one.

In view of Theorem 4, we must prove only the necessity of the condition on $\mathrm{gcd}$. Proof [1], [5].

Suppose $d>1$ is the greatest common divisor of lengths of all cycles of $\Gamma$. Let us consider a tree $T$ with root $\mathbf{p}$ and with all vertices of the graph. Suppose $t(\mathbf{p})=0$ and for every edge $\mathbf{r} \rightarrow \mathbf{q}$ of the tree suppose $t(\mathbf{r})=t(\mathbf{q})+1$ (modulo $d)$. So $t(\mathbf{q})<d$ for every vertex $\mathbf{q}$.

Let the edge $\mathbf{u} \rightarrow \mathbf{v}$ be outside of $T$. If $t(\mathbf{u}) \neq t(\mathbf{v})+1$ (modulo d) then two paths from $\mathbf{p}$ to $\mathbf{v}$ through the edge $\mathbf{u} \rightarrow \mathbf{v}$ and the edges of $T$ and through only the edges of $T$ have not equal (modulo $d$ ) lengths. Therefore in strongly connected graph $\Gamma$ there are two cycles having not equal lengths (modulo $d$ ). It contradicts to the choice of $d$ as $g c d$ of lengths of all cycles.

So for any edge $\mathbf{u} \rightarrow \mathbf{v}$ one has $t(\mathbf{u})=t(\mathbf{v})+1$ (modulo $d$ ). Consequently by whatever coloring for any word $s$ of the colors one has $t(\mathbf{u} s)=t(\mathbf{v} s)+1$. So any word $s$ could not unite $\mathbf{v}$ and $\mathbf{u}$, whence $\Gamma$ has no synchronizing coloring.

Let us recall that the vertex $\mathbf{q}$ of the graph $\Gamma$ is called a sink if there exists a way on $\Gamma$ from any vertex to $\mathbf{q}$.

Theorem 6 A finite directed graph $\Gamma$ with constant outdegree of all its vertices has synchronizing coloring if and only if $\Gamma$ has a sink and in the strongly connected component $H$ of the sink the greatest common divisor of lengths of all cycles is one.

Proof. The necessity of a sink is obvious, the necessity of conditions on $H$ follows from Theorem 5 because any subgraph of $\Gamma$ has synchronizing coloring.

Let us go to the sufficiency. There exists in $\Gamma$ a tree $T$ with root in sink. Let us give all edges from $T \backslash H$ common color $\alpha$. Therefore the word $\alpha^{i}$ for some $i$ maps $\Gamma$ on $H$. So the proof is reduced to the conditions of Theorem 5 . 


\section{References}

1. R.L. Adler, L.W. Goodwyn, B. Weiss. Equivalence of topological Markov shifts, Israel J. of Math. 27, 49-63, 1977.

2. R.L. Adler, B. Weiss. Similarity of automorphisms of the torus, Memoirs of the Amer. Math. Soc. 98, Providence, RI, 1970.

3. G. Budzban, A. Mukherjea. A semigroup approach to the Road Coloring Problem, Probability on Algebraic Structures. Contemporary Mathematics, 261, 195-207, 2000.

4. A. Carbone. Cycles of relatively prime length and the road coloring problem, Israel J. of Math., 123, 303-316, 2001.

5. K. Culik II, J. Karhumaki, J. Kari. A note on synchronized automata and Road Coloring Problem, Developments in Language Theory (5th Int. Conf., Vienna, 2001), Lecture Notes in Computer Science, 2295, 175-185, 2002.

6. J. Friedman. On the road coloring problem, Proc. of the Amer. Math. Soc. 110, 1133-1135, 1990 .

7. E. Gocka, W. Kirchherr, E. Schmeichel, A note on the road-coloring conjecture. Ars Combin. 49, 265-270, 1998.

8. R. Hegde, K. Jain, Min-Max theorem about the Road Coloring Conjecture EuroComb 2005, DMTCS proc., AE, 279-284, 2005.

9. N. Jonoska, S. Suen. Monocyclic decomposition of graphs and the road coloring problem, Congressum numerantium, 110, 201-209, 1995.

10. J. Kari. Synchronizing finite automata on Eulerian digraphs, Springer, Lect. Notes in Comp. Sci., 2136, 432-438, 2001.

11. P. Lankaster. Theory of Matrices, Acad. Press, NY - London, 1969.

12. D. Lind, B. Marcus. An Introduction of Symbolic Dynamics and Coding, Cambridge Univ. Press, 1995.

13. A. Mateescu, A. Salomaa, Many-Valued Truth Functions, Černy's Conjecture and Road Coloring, Bull. of European Ass. for TCS, 68, 134-148,1999.

14. G.L. O'Brien. The road coloring problem, Israel J. of Math., 39, 145-154, 1981.

15. D. Perrin, M.P. Schǔtzenberger. Synchronizing prefix codes and automata, and the road coloring problem, In Symbolic Dynamics and Appl., Contemp. Math., 135, 295-318, 1992.

16. J.E. Pin. On two combinatorial problems arising from automata theory, Annals of Discrete Math., 17, 535-548, 1983.

17. A.N. Trahtman. Notable trends concerning the synchronization of graphs and automata, CTW06, El. Notes in Discrete Math., 25, 173-175, 2006. 